\title{
Cerebello-Pontine Angle Glioblastoma with Cervical Spine Metastasis: A Case Report
}

\author{
Giuseppe Mariniello, MD", Carmela Peca, MD', Maria Laura Del Basso De Caro, MD², \\ Sergio Corvino, $M D^{1}$, Valentina Orlando, $M D^{1}$, Elia Guadagno, $M D^{2}$ and Francesco \\ Maiuri, $M D^{1^{*}}$
}

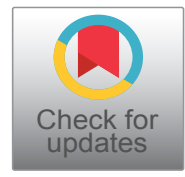

${ }^{1}$ Department of Neurosciences and Reproductive and Odontostomatological Sciences, Neurosurgical Clinic, University of Naples Federico II, Naples, Italy

${ }^{2}$ Department of Advanced Biomorphological Sciences, University of Naples Federico II, Naples, Italy

*Corresponding author: Francesco Maiuri, MD, Department of Neurosciences and Reproductive and Odontostomatological Sciences, Neurosurgical Clinic, University of Naples Federico II, Naples, Italy, Tel: +39 81-7462581, Fax: +39 81-7462594

\begin{abstract}
This article reports a left cerebellopontine angle glioblastoma presenting with two week history of rapidly progressive hearing loss and trigeminal pain, treated by subtotal resection and radiotherapy with concomitant and adjuvant temozolomide. At one year, local tumor control and diffuse neoplastic seeding in the cervical spinal cord were evidenced.

Glioblastomas involving the cerebellopontine angle are exceptional with only 10 reported cases. The possibility of a glioblastoma should be considered in the differential diagnosis of malignant cerebellopontine angle tumors. The standard treatment including maximum tumor resection and Stupp protocol is justified to obtain local tumor control.
\end{abstract}

\section{Keywords}

Glioblastoma, Cerebellopontine angle tumor, Spinal cord metastasis

\section{Introduction}

Primary infratentorial glioblastomas are uncommon in adult patients and are found in the cerebellum (1.5\%) or brainstem (4.1\%) [1]. The cerebellopontine angle is an exceptional location of glioblastoma, with only 10 reported cases [2-11]. Six of them were intra-axial, arising from the cerebellum $[3,7,9]$ or pons $[2,4,6]$, with lateral exophytic growth.

Three cases $[5,10,11]$ were primary extra-axial glioblastomas of the cerebellopontine angle arising from the root entry zone of the eighth cranial nerve, whereas in one [8] the origin was not defined.

This report describes a case of glioblastoma of the cerebellopontine angle, with local tumor control and diffuse neoplastic seeding of the cervical spinal cord during the follow-up.

\section{Case Description}

A 49-year-old female patient was observed because of a two-week history of dizziness, rapidly progressive left deafness and trigeminal pain in V2 and V3 on the left side. Some days before admission, headache and gait instability also occurred. At neurological examination complete hearing loss on the left side, nystagmus on the lateral eye movements, left facial hypoesthesia and slight ataxia were found. The audiometric test confirmed the finding of complete left anacusia.

Magnetic resonance imaging (Figure 1) revealed a tumor mass in the left cerebellopontine angle, involving left cerebellar hemisphere and the cisternal portion of the VII and VIII cranial nerves. It was hypointense in T1 weighted sequences and hyperintense in T2 weighted and FLAIR sequences, and showed marked and homogenous enhancement.

At surgery, the left cerebellopontine angle was exposed through left suboccipital retrosigmoid approach. A grey-reddish, moderately vascularized tumor was found in the cerebellopontine angle. It mainly grew 

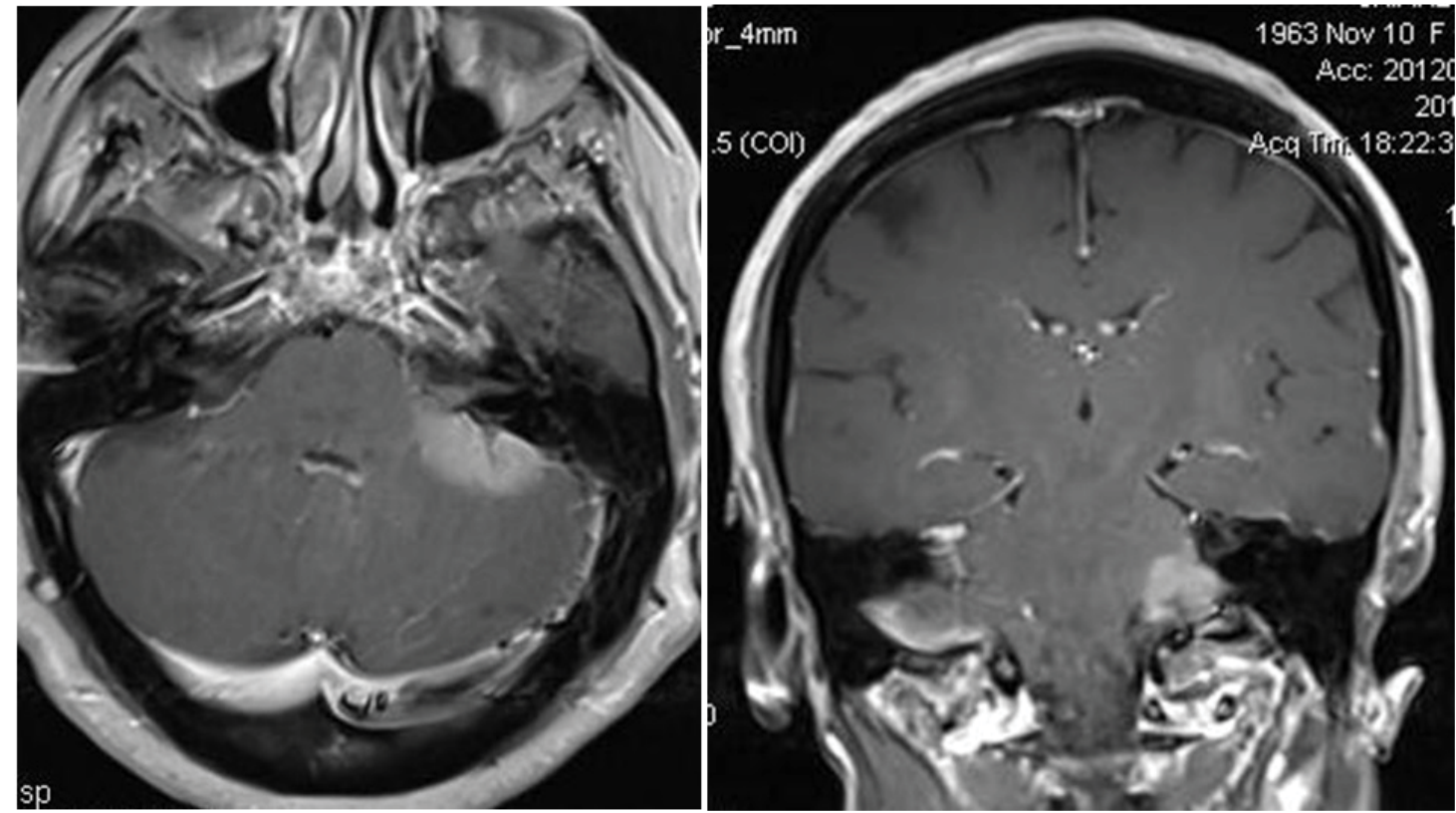

Figure 1: Magnetic resonance, post-contrast T1-weighted axial a) Coronal; b) Sequences: Left cerebellopontine angle tumor involving the cerebellar hemisphere and the left acoustic and facial nerves.

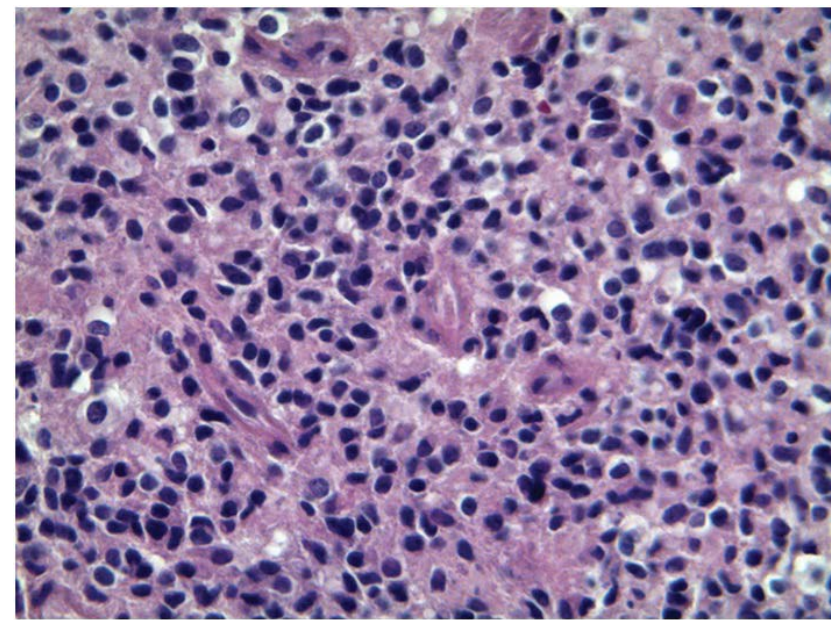

Figure 2: Histological specimen: The neoplasm is composed by a highly cellular proliferation of small cells with round and hyperchromatic nuclei and minimal discernible cytoplasm and with brisk mitotic activity (H-E 40x).

in the cisternal space involving the acoustic and facial nerves, and also infiltrated the pia and cortex of the lateral cerebellar surface. Intraoperative histology of a tumor specimen was in favour of high grade glioma. Large tumor resection was made by microsurgical technique by sparing the cranial nerves. Locoregional chemotherapy with carmustine wafers was also administered.

The histopathological specimen (Figure 2) showed a highly cellular tumor with small cells, with hyperchromatic nuclei and minimal discernible cytoplasm. The immunohistochemical staining of tumor cells revealed GFPA expression, proliferation index Ki67 Li 30\% and negative IDH expression (IDH wild type). The final diagnosis was glioblastoma (WHO IV).

Postoperatively, slight and transient left facial paresis and remission of the trigeminal pain were observed.
Radiotherapy with concomitant and adjuvant temozolomide, according to the Stupp [12] protocol, was administered. Magnetic resonance studies at 3 and 6 months showed no residual tumor.

One year after surgery the patient complained of dizziness and gait difficulty. Cranial magnetic resonance (Figure 3a) showed enhancement of the left VII and VIII cranial nerves with no tumor recurrence. Magnetic resonance of the cervical spine (Figure $3 b$, Figure $3 c$ and Figure $3 d$ ) evidenced diffuse neoplastic seeding on the posterior and left lateral aspect of the cervical spinal cord. Second line chemotherapy with fotemustine was administered. The patient died 3 months later.

\section{Discussion}

This case is noteworthy for several features, including the exceptional location in the cerebellopontine angle (with 10 reported cases), the clinical onset with rapidly progressive hearing loss and trigeminal pain, and the subsequent tumor seeding in the cervical spinal cord, inspite of the tumor control at the initial location.

Among the 10 reported cases [2-11] (Table 1 ) seven were men and three women; three of them were very young (22-years-old or less). Six were exophytic tumors arising from the cerebellum $[3,7,9]$ or pons $[2,4,6]$ and three $[5,10,11]$ arose from the eighth cranial nerve (likely from the nervous tissue of its proximal part or from neuroglial cells in the surrounding leptomeninges).

The clinical history before diagnosis was very short (from one hour to the three months) in all but one cases. The neurological symptoms were those of unilateral involvement of the cranial nerves of the cerebellopontine angle, mainly hearing loss and facial paresis; ataxia and intracranial hypertension were also 






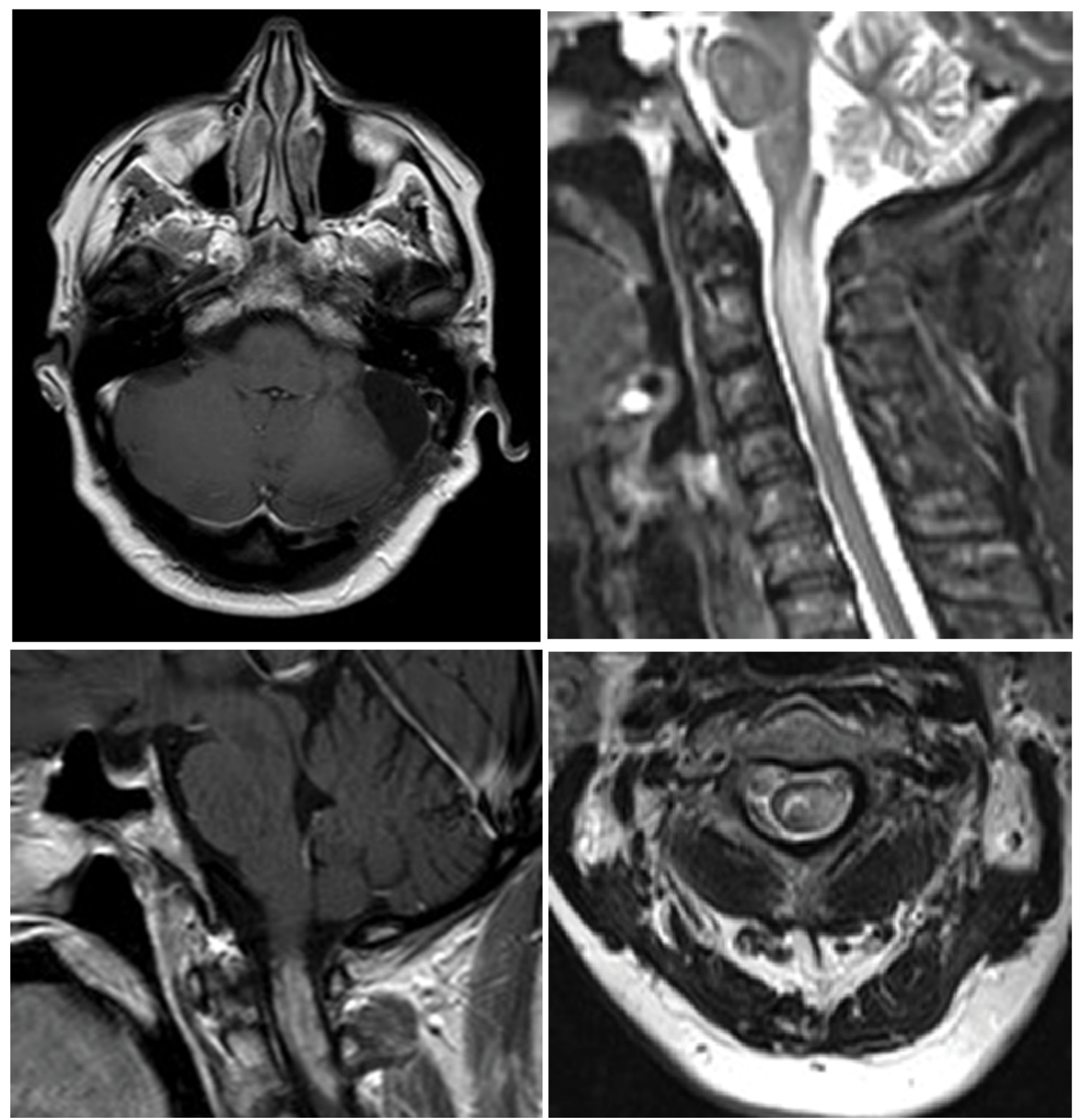

Figure 3: Cranial and cervical post-contrast magnetic resonance, one year after surgery. a) Cranial axial image, T1-weighted sequence: No tumor recurrence; b-d) Cervical images; b) T2-weighted sagittal sequence; c,d) T1-weighted sagittal; c) Axial; d) Sequences after contrast administration: Diffuse neoplastic seeding on the posterior and lateral aspects of the cervical spinal cord up to C3.

\section{referred.}

The preoperative neuroradiological diagnosis is difficult. Some reported cases were misdiagnosed as benign extra-axial tumors, such as meningioma $[5,8]$ or schwannoma $[2,11]$; in others $[7,9,10]$ a malignant tumor was suspected from several MRI findings, including intratumoral necrosis, heterogeneous enhancement, peritumoral edema, thickening and enhancing eighth cranial nerve, high choline peak on MRI spectroscopy. However, the correct radiological diagnosis of glioblastoma was never made preoperatively.

The best management of cerebellopontine angle glioblastomas is not well established, because of their exceptional occurrence. Nine patients underwent subtotal resection of the tumor mass in order to preserve the cranial nerves; only one underwent stereotactic biopsy for tumor diagnosis. The adjuvant radiotherapy was administered in 5 patients.
The outcome of cerebellopontine angle glioblastoma is poor. Four among 8 patients whose outcome is specified died within 3 months after surgery and only 2 were still alive at one and two years respectively, as in our case. This may be explained by the difficulty of gross total resection and the extra-axial cisternal location with increased risk of leptomeningeal dissemination.

In the present case an extensive leptomeningeal diffusion from the initial tumor site along the cervical spinal canal, with involvement of the cervical spinal cord occurred one year after surgery. This was responsible for the rapid clinical worsening and death, in spite of the absence of tumor regrowth at the initial site.

\section{Conclusion}

A short clinical history with rapidly progressive symptoms and signs of unilateral seventh and eighth cranial nerves should suggest a malignant cerebellopontine an- 
gle tumor. The magnetic resonance must focus the diagnosis of glioblastoma on spectroscopy and perfusional sequences. Maximum tumor resection followed by radiotherapy and temozolomide should be used also for this peculiar location. However, the best management will be defined from a larger number of cases.

\section{References}

1. Larjavaara S, Mantyla $R$, Salminen $T$, Haapasalo $H$, Raitanen J, et al. (2007) Incidence of gliomas by anatomic location. Neuro Oncol 9: 319-325.

2. Swaroop GR, Whittle IR (1997) Exophytic pontine glioblastoma mimicking acoustic neuroma. J Neurosurg Sci 41: 409-411.

3. Kasliwal MK, Gupta DK, Mahapatra AK, Sharma MC (2008) Multicentric cerebellopontine angle glioblastoma multiforme. Pediatr Neurosurg 44: 224-228.

4. Rasalingam K, Abdullah JM, Idris Z, Pal HK, Wahab N, et al. (2008) A rare case of paediatric pontine glioblastoma presenting as a cerebellopontine angle otogenic abscess. Malays J Med Sci 15: 44-48.

5. Wu B, Liu W, Zhu H, Feng H, Liu J (2011) Primary glioblastoma of the cerebellopontine angle in adults. $J$ Neurosurg 114: 1288-1293.
6. Salunke P, Sura S, Tewari MK, Gupta K, Khandelwal NK (2012) An exophytic brain stem glioblastoma in an elderly presenting as a cerebellopontine angle syndrome. $\mathrm{Br} \mathrm{J}$ Neurosurg 26: 96-98.

7. Matsuda M, Onuma K, Satomi K, Nakai K, Yamamoto $\mathrm{T}$, et al. (2014) Exophytic cerebellar glioblastoma in the cerebellopontine angle: Case report and review of the literature. J Neurol Surg Rep 75: e67-e72.

8. Lee JH, Kim JH, Kwon TH (2017) Primary glioblastoma of the cerebellopontine angle: Case report and review of the literature. J Korean Neurosurg Soc 60: 380-384.

9. Panigrahi S, Mishra SS, Das S (2017) Primary cerebellopontine angle glioblastoma in an adult. Asian $\mathrm{J}$ Neurosurg 12: 62-64.

10. Takami H, Prummer CM, Graffeo CS, Peris-Celda M, Giannini C, et al. (2018) Glioblastoma of the cerebellopontine angle and internal auditory canal mimicking a peripheral nerve sheath tumor: Case report. J Neurosurg 21: 1-5.

11. Yang DX, Jing Y, Xu ZM, Yuan F, Liu YL (2018) Primary glioblastoma of cerebellopontine angle in adult mimicking acoustic neuroma. World Neurosurgery 122: 48-52.

12. Stupp R, Warren $P$ Manson, Martin $J$ van den Bent, Michael Weller, Fisher B, et al. (2005) Radiotherapy plus concomitant and adjuvant temozolomide for glioblastoma. N Engl J Med 352: 987-996. 